[DOI: 10.24214/jecet.A.10.2.15358. ]

Juurnal of Enviranmental Science, Computer Science and Engineering \& Technology

An International Peer Review E-3 Journal of Sciences and Technology

Available online at www.jecet.org

Section A: Environmental Science

Research Article

\title{
Research on Charging Infrastructure Planning Based on Kernel Density Analysis
}

\author{
Chundong Li, Tongqing Zhang, Xiaochuan Tan, Xingbang Gao, Dong Guo* \\ School of Transportation and Vehicle Engineering Shandong University of Technology China, \\ Shandong, Zibo, Zhangdian, 255049
}

Received: 07 March 2021; Revised: 18 March 2021; Accepted: 28 March 2021

\begin{abstract}
In order to effectively solve resource and environmental problems, the market of new energy vehicles, represented by electric vehicles, is growing rapidly. The convenience of charging infrastructure layout is an important factor affecting the development of electric vehicles, so how to reasonably add charging infrastructure has become a key issue to be solved. Starting from geography and planning, this paper uses kernel density analysis to explore the spatial distribution of different types of commercial service facilities in Zibo city, and obtains the suitable area for adding charging piles, so as to put forward suggestions for adding charging infrastructure in Zibo.
\end{abstract}

Keywords: ArcGis; charging infrastructure; kernel density analysis; point of interest

\section{INTRODUCTION}

The "China Urban Energy Report" shows that China's current transportation energy consumption accounts for $10 \%$ of the country's total terminal energy consumption, of which oil consumption accounts for $37 \%$ of the country's terminal oil consumption. In order to alleviate the environmental pollution and energy consumption caused by the demand for motor vehicles, China has taken pure electric vehicles as the main strategic orientation of the transformation of the automobile industry. Based on relevant policy support, the annual growth rate of electric vehicle ownership in Cnina will not be less than 30\%. By June 2020, the number of new energy vehicles in China has reached 3.404 million, while the charging infrastructure has reached 1.382 million. There is still a big gap between this data 
and the ratio between electric vehicles and charging infrastructure will reach $1: 1^{[1]}$. The increasing load of electric vehicles leads to an increase in the amount of charging infrastructure required by them. However, the construction of charging infrastructure requires a large amount of land resources. In cities with limited space, how to use the existing land to add charging infrastructure has become an urgent problem to be solved.

A large number of scholars have studied the kernel density analysis. For example, Feng Daici introduced kernel density estimation based on POI data to analyze the spatial aggregation degree of commercial service facilities in Shenzhen ${ }^{[2]}$.Li Juan et al. used kernel density estimation to analyze the layout of public parking lots in cities, providing reference for parking lot planning ${ }^{[3]}$.Shi Beixiang et al. used kernel density estimation to study the spatial form of large-scale cities, and summarized the spatial form characteristics of large-scale cities ${ }^{[4]}$.

In this paper, kernel density analysis is conducted on the data of different types of POI in the city to obtain the spatial distribution of different types of service facilities in the study area, identify the agglomeration of different types of service facilities and the distribution of hot spots, and provide a theoretical basis for the rational use of the existing land to add charging infrastructure.

\section{THE METHODS}

Kernel density analysis method is based on the first law of geography, that is, the closer the distance is, the more closely related things are, and the closer the location is to the core elements, the greater the density expansion value is obtained, which reflects the difference of spatial location and the attenuation of center strength with distance, and is suitable for density estimation of geographical phenomena such as the influence of urban facilities and services. Therefore, this study uses the kernel density method to realize POI facility service impact diffusion.The formula of kernel density is defined as

$$
f(s)=\sum_{i=1}^{n} \frac{1}{h^{2}} \lambda\left(\frac{s-c_{i}}{h}\right)
$$

Where, $f(s)$ is the kernel density estimation function at position $s$, and $h$ is the path attenuation threshold, that is, bandwidth; $c_{i}$ is the spatial position of the $i$-th POI point in the bandwidth; $\mathrm{n}$ is the number of POI points whose path distance from position $s$ is less than or equal to $h$; the $\lambda$ function is a spatial weight function. In this paper, the quaternary weight equation is selected, and the formula is ${ }^{[5]}$

$$
\lambda\left(\frac{s-c_{i}}{h}\right)=\frac{3}{4}\left[1-\left(\frac{s-c_{i}}{h}\right)^{2}\right]
$$

By considering the equilibrium distribution of different POI interest points, the calculation formula of path attenuation threshold, namely bandwidth, in this paper is as follows

$$
\begin{aligned}
& h=0.9 \times \min \left(S D, \sqrt{1 / \ln 2} \times D_{m}\right) \times N^{-2} \\
& S D=\sqrt{\left[\sum_{i=1}^{N}\left(x_{i}-\bar{X}\right)^{2}\right] / N+\left[\sum_{i=1}^{N}\left(y_{i}-\bar{Y}\right)^{2}\right] / N}
\end{aligned}
$$


Where, $S D$ is the standard distance between the average center of the input point and all points; $D_{m}$ is the median distance between the average center of the input point and all points. $x_{i}$ and $y_{i}$ are the coordinates of $i ;(X, Y)$ represents the average center of input points; $N$ is the total number of elements. Through the kernel density analysis, this paper can directly reflect the density of service facilities and the scope of spatial influence, and comprehensively reflect the spatial form.

\section{CASE ANALYSIS}

A. Overview of the study area: Zibo City as a typical industrial city, its unique "group type" urban layout is known as the "Zibo Model" by experts. Zibo has 5 districts and 3 counties, with a total area of 5,965 square kilometers. The built-up area covers an area of 341.9 square kilometers. It has 30 streets and 58 towns under its jurisdiction, with an urban rate of $72.04 \%$.

B. The data type: POI (Point of Interest) data mainly handle four aspects of information: name, address, coordinate and classification. This paper takes the road network data of Zibo City before September 2020 and the interest points of service facilities in Zibo City as the data sources, and collects the POI data of Zibo City on AMAP through the POI collector of AMAP.

In this paper, according to the classification standard in "Code for Classification of Urban Land Use and Planning Standards of Development Land " GB50137-2011 ${ }^{[6]}$, the POI data obtained in Zibo City is divided into 8 categories, and the specific classification is shown in the following Table 1.

Table 1: The percentage of different types of POI data

\begin{tabular}{cccc}
\hline Number & Types & Data & Proportion \\
\hline 1 & Residential Area & 3101 & $9.39 \%$ \\
2 & Supermarket & 6098 & $18.47 \%$ \\
3 & Education & 6794 & $20.57 \%$ \\
4 & Company & 6795 & $20.58 \%$ \\
5 & Sport and Leisure & 2453 & $7.43 \%$ \\
6 & Parking Lot & 2866 & $8.68 \%$ \\
7 & Gas Station & 498 & $1.51 \%$ \\
8 & Bus Station & 4419 & $13.38 \%$ \\
\hline
\end{tabular}

C. Status analysis of different types of POI: In ArcGIS, through kernel density analysis of points of each POI category, the spatial distribution of interest points of different POI is analyzed, and then the modification suggestions are put forward for adding charging piles at different service facility points.

Fig. 1 is the distribution diagram of all types of POI data points in Zibo City. Kernel density analysis was conducted on all POI data points to obtain a visual three-dimensional view of kernel density analysis, as shown in Fig. 2.As can be seen from Figure 2, the high-density areas obtained by kernel density analysis are mainly concentrated in the 8 districts and counties of Zibo City. Due to the large number of data, this paper takes Zhangdian District as an example for analysis. 


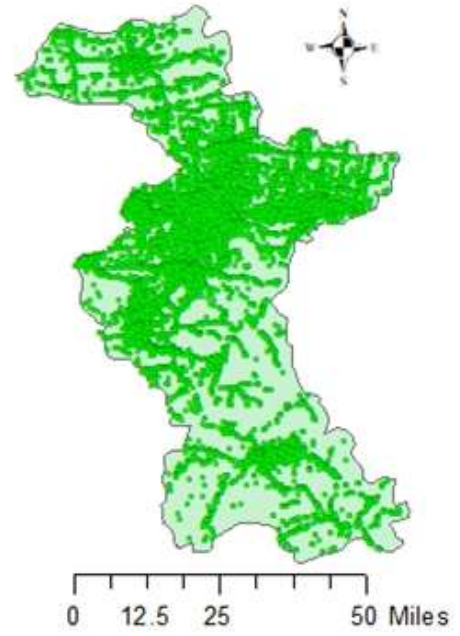

Fig. 1: Distribution diagram of all POI data in Zibo

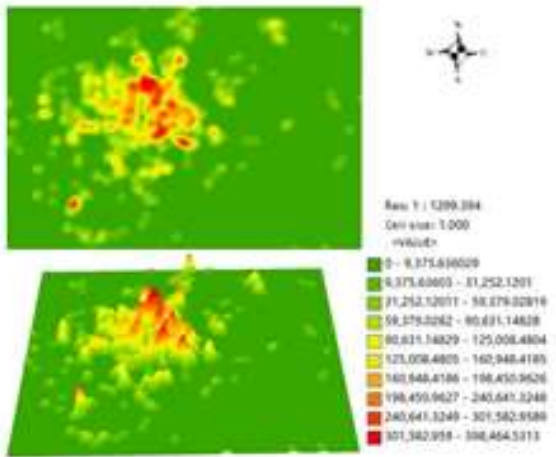

Fig. 3: Schematic diagram of kernel density analysis of residential area
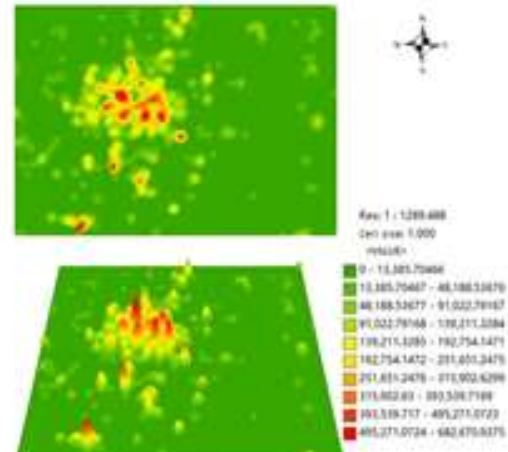

Fig. 5: Schematic diagram of kernel density analysis of the education

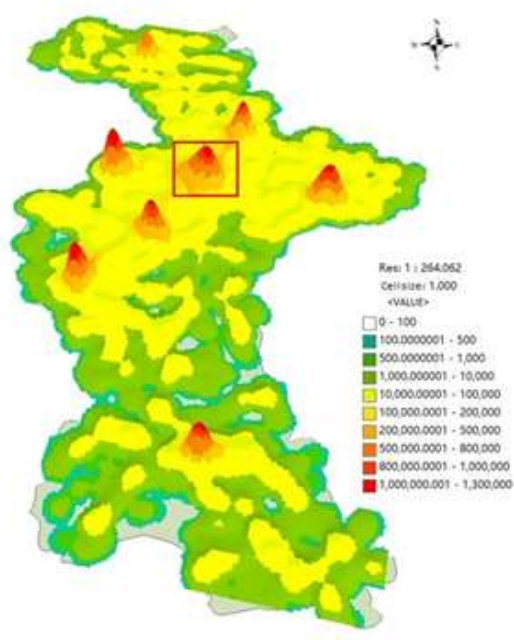

Fig. 2: Kernel density analysis diagram of all POI data points in Zibo

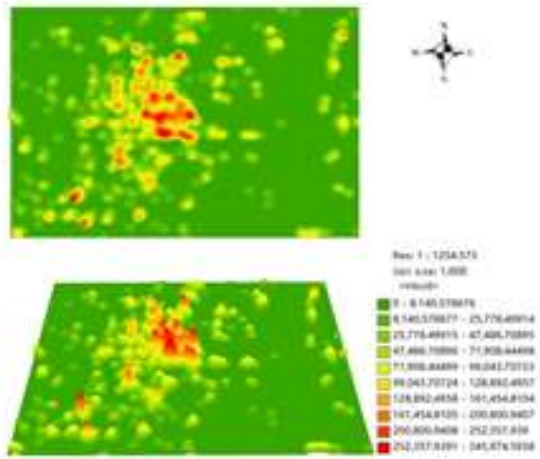

Fig. 4: Schematic diagram of kernel density analysis of supermarket

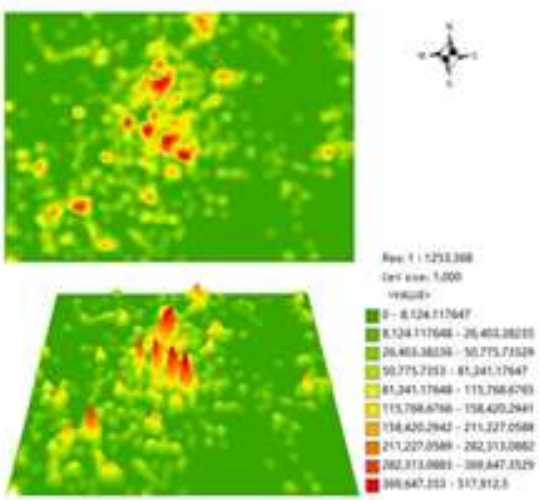

Fig. 6: Schematic diagram of kernel density analysis of the company 


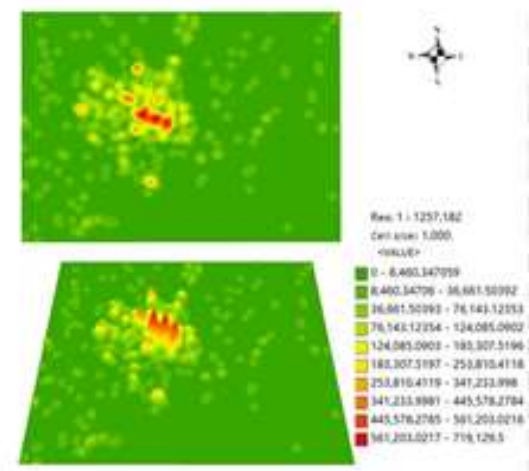

Fig. 7: Schematic diagram of kernel density analysis of sports and leisure

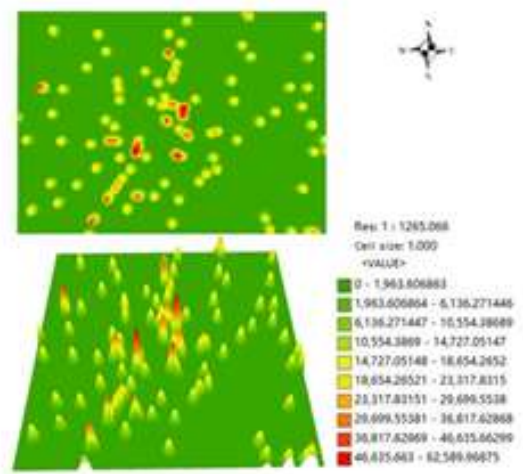

Fig. 9: Schematic diagram of kernel density analysis of gas station

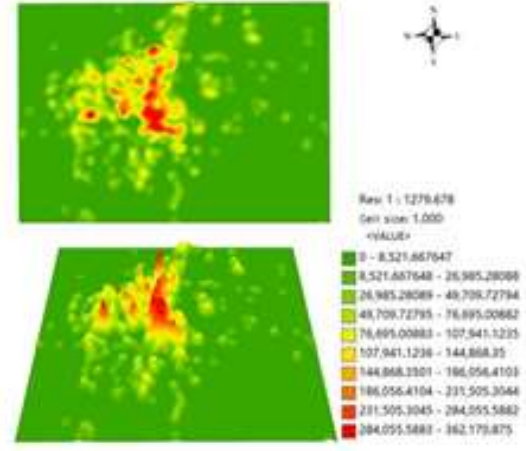

Fig. 8: Schematic diagram of kernel density analysis of parking lot

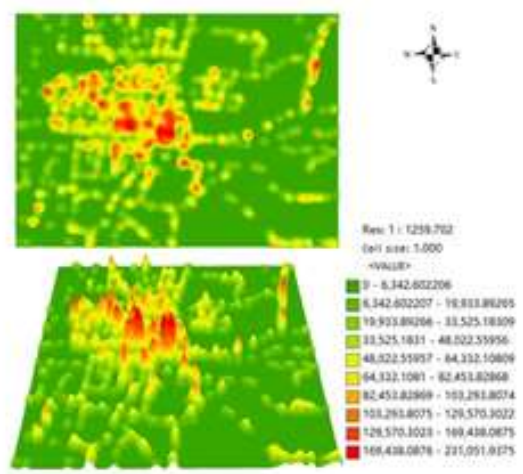

Fig. 10: Schematic diagram of kernel density analysis of bus station

Fig.3-10 shows the kernel density visualization analysis of different types of POI data in Zhangdian District. According to the visualization analysis diagram, except for gas stations and bus stations, the distribution of other POI interest points presents the characteristics of contiguous aggregation, with high spatial density. To this end, a general summary is made for different types of locations:

1. The parking space within the neighborhood can be used to transform the charging infrastructure, and the green space within the neighborhood can be used to add charging piles on the premise of ensuring the green land rate.

2. Charging piles can be added in the underground space near the supermarket, and resources can be efficiently utilized based on the Internet of Things.

3. Schools can use the parking space on campus to transform the original parking space and implement the Internet of Things technology to maximize the utilization of resources.

4. Road planning can be carried out around industrial parks and other areas, and charging piles can be set up in advance with full consideration of the future use of charging piles.

5. Gas stations and other sites can make use of the underground space and the remaining space on the ground to add charging pile parking Spaces on the premise of safety. 


\section{CONCLUSION}

Electric vehicles are the trend of green environmental protection in the future. In the future, charging piles should provide better services for ordinary people, facilitate the approval and use of land, establish a sound management system, and ensure the safe and efficient operation of charging service network. With the help of POI geospatial big data, this paper obtained the distribution characteristics and aggregation degree of different types of service facilities in Zibo City by using kernel density analysis, and put forward suggestions and suggestions for reasonable addition of charging infrastructure in Zibo City based on the policy.

\section{REFERENCES}

1. National Development and Reform Commission of the people's Republic of China. Guidelines for the development of electric vehicle charging infrastructure (2015-2020) [R/OL]. 2015-1009. Available: http://www.ndrc.gov.cn/zcfb/zcfbtz/201511/t20151117_758762.html.

2. Daici Feng, Commercial Spatial Pattern Analysis in Shenzhen Based on POI Data. Wuhan University. May,2018.

3. Juan Li and Yang Yu. Research on the Spatial Distribution of Public Parking Lots in Mingcheng District of Xi 'an Based on Kernel Density and Accessibility [J].Urban Architecture, 2019,16 (01) : 13-16.

4. Beixiang Shi and Junyan Yang. Spatial Morphology Analysis of Large Scale Based on GIS Platform: A Case Study of Height, Density and Intensity of Megacities [J].Urban Planning International, 2019,34 (02) : 111-117.

5. Chen Fei and Daosheng Du, Application of the Integration of Spatial Statistical Analysis with GIS to the Analysis of Regional Economy. Wuhan University. May,2018.

6. GB 50137-2011, Urban land classification and standard for planning and construction land [S]. Beijing: Ministry of housing and urban rural development of the people's Republic of China, 2012.

\section{* Corresponding Author: Dong Guo,}

School of Transportation and Vehicle Engineering Shandong University of Technology China, Shandong, Zibo, Zhangdian, 255049

Online date of publication: 30.03 .2021 